http://jmscr.igmpublication.org/home/

ISSN (e)-2347-176x ISSN (p) 2455-0450

crossref DOI: https://dx.doi.org/10.18535/jmscr/v9i7.32

\author{
Dournal Of Medical Science And Clinical Research \\ IGM Publication \\ An Official Publication of IGM Publication
}

\title{
Chondro Osseous Respiratory Epithelial Adenomatoidhamartoma
}

\author{
Authors \\ Dr Alisha Teresa Jose ${ }^{1}$, Dr Lillykutty Pothen ${ }^{2}$, Dr Sankar $\mathbf{S}^{3}$ \\ ${ }^{1}$ Junior Resident, Department of Pathology, Government Medical College Kottayam, Kerala \\ ${ }^{2}$ Additional Professor, Department of Pathology, Government Medical College Kottayam, Kerala \\ ${ }^{3}$ Professor and HOD, Department of Pathology, Government Medical College Kottayam, Kerala
}

\begin{abstract}
COREAH is a benign lesion of nasal cavity and paranasal sinuses which is extremely rare with only a few cases reported in the literature till date. We herein report an infrequent case of COREAH in a 47 year old male who presented with nasal obstruction and was found to have a choanal mass. The mass was completely excised and sent for histopathological examination. Microscopy showed hamartomatous proliferation of respiratory type glands with numerous spicules of mature bone and cartilage characteristic of COREAH. COREAH is of importance because it can be clinically mistaken for wide range of both benign and malignant neoplasm. Identification of this lesion as a benignenityis particularly important due to its potentially worrisome radiographic appearance and to avoid unwanted radical surgical procedures.

Keywords: Nasal cavity, Hamartoma.
\end{abstract}

\section{Introduction}

Hamartomas of the nasal cavity are extremely rare, usually presenting as polypoid mass, nasal obstruction and epistaxis. They usually consist of mesenchyme and may also include respiratory epithelial elements. Hamartomas of the sinonasal tract include Respiratory Epithelial Adenomatoid Hamartoma, seromucinous hamartoma, Chondromesenchymal hamartoma and chondroosseus respiratory epithelial adenomatoid hamartoma. ${ }^{(1)}$

Although these lesions are benign they can grow to relatively larger sizes and may pose worrisome clinical picture. Also radiographically they may appear aggressive. We report herein a rare case of COREAH of the nasal cavity emphasising its clinical and pathological features and the importance of recognising and differentiating it from other more aggressive lesions of the nasopharynx. ${ }^{(2)}$

\section{Case Report}

A 47 year old male presented with nasal obstruction of a few months duration; however no epistaxis, rhinorrhoea or hyposmia was reported. CECT was performed which showed a partially calcified mass in posterior choana extending into nasopharynx. Initial endoscopic biopsy obtained was reported as inflammatory polyp. Later MRI revealed a well defined heterogeneous $\mathrm{T} 2$ signal moderately enhancing lesion in the right nasal cavity with lobulated margins extending from right middle meatus into nasopharynx. Moderate heterogenous post enhancement noted. Diagnostic 
nasal endoscopy revealed a bony hard swelling filling posterior end of choana and nasopharynx. Endoscopic removal of the mass was done and sent for histopathological examination

We received 2 nodular tan white soft tissue pieces measuring $3.5 \times 2.5 \times 2 \mathrm{~cm}$ and $2.8 \times 2 \times 1 \mathrm{~cm}$. cut section was whitish glistening. Microscopic section showed a lesion lined by respiratory epithelium with mild invagination into subepithelium with focus of squamous metaplasia. Subepithelium shows proliferation of seromucinous glands with islands of cartilage and bony tissue seen scattered in a fibrous stroma.

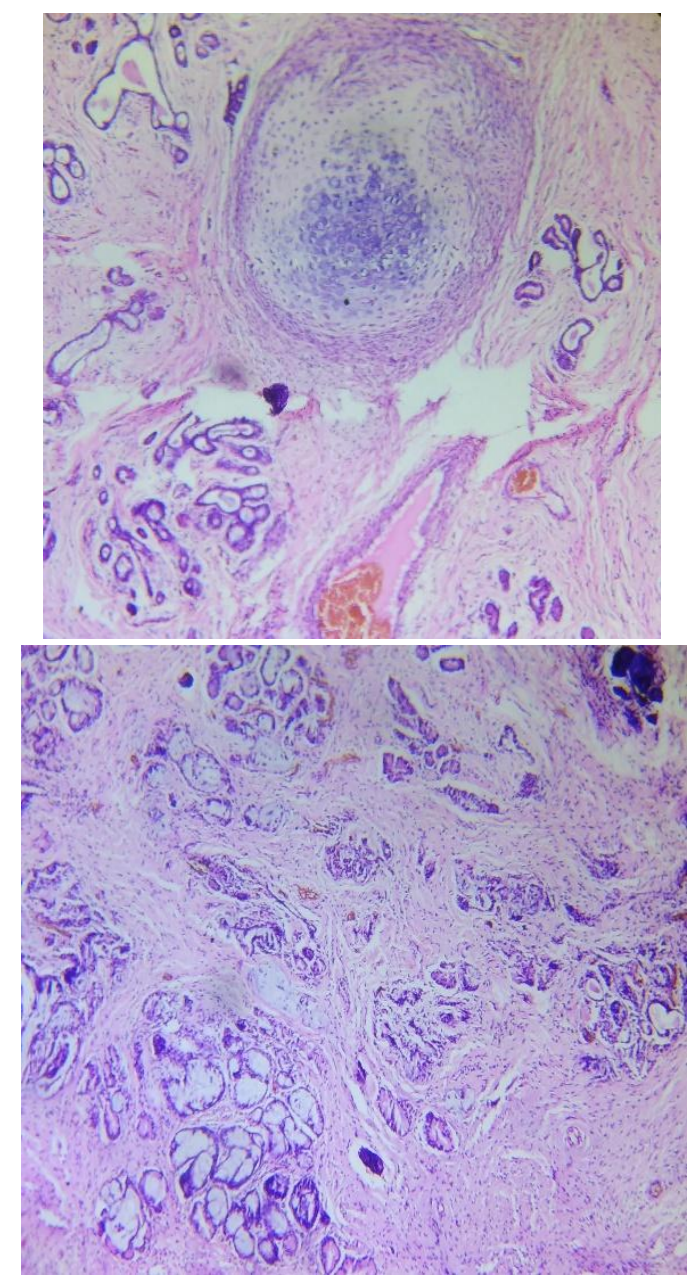

Fig 1.0 Biopsy no: 6843/2019: Endoscopic biopsy showing proliferation of seromucinous glands and islands of cartilage

\section{Discussion}

Albrecht coined the term hamartoma in 1904 which emanates from greek hamartia denoting error and oma benign growth. Hamartoma is defined as disorganised but benign mass composed of cells indigenous to the involved site. It neither indicates a neoplastic or an inflammatory process nor have the capacity for continuous unimpeded growth. Hamartomas of the head and neck in general and sinonasal tract in particular are extremely rare. Until the early 1990s the majority of the sinonasal hamartomas were reported to be mesenchymal, most frequently vascular in origin. However epithelial hamartomas namely REAHs have since been more frequently reported. ${ }^{(1)}$

REAH has long been identified as an exclusively epithelial hamartoma of sinonasal tract while nasal chondromesenchymal hamartoma has been identified as a mesenchymal hamartoma. COREAH has been proposed to lie in spectrum between REAH and NCMH as it includes both epithelial and mesenchymal elements.

$\mathrm{NCMH}$ was first described in 1998 by MC Dermott et al in a case series of 7 patients. NCMH has been regarded as the upper respiratory tract homolog of chest wall mesenchymal hamartoma. REAH was first described by Wenig and Heffner in 1995. Nasal cavity accounts for $70 \%$ of REAH. REAH is a benign acquired overgrowth of indigenous glands of the sinonasal tract arising from the surface epithelium. Clinically, REAH are noted to arise in the nasal cavity in particular the posterior nasal septum, the other sites being lateran nasal wall, middle meatus and inferior turbinate. They usually present with nasal obstruction, stuffiness, epistaxis and chronic rhinosinusitis. Seromucinous or glandular hamartomas consist of epithelial proliferation of both small and large glands arranged in clusters or lobules .larger glands are lined by ciliated respiratory epithelium while the small glands are lined by flat to cuboidal epithelium. They are distinguished from COREAH by the presence of proliferating serous acini and absence of chondro osseous metaplasia. ${ }^{(1,2)}$

Clinical differential diagnosis for RHEA and COREAH include inflammatory polyps, inverted pappillomas and low grade sinonasal 


\section{JMSCR Vol||09||Issue||07||Page 191-193||July}

adenocarcinoma. Inflammatory polyps can be distinguished from COREAH both clinically and microscopically. While COREAH are solitary and usually arising in posterior nasal cavity, inflammatory polyps are bilateral and and mostly arise in the middle meatus. Inflammatory polyps are associated with inflammation, allergy, or mucoviscidosis. Histologically it is characterised by loose myxoid or hyaline stroma covered by respiratory epithelium without adenomatoid proliferation.

Inverted papillomas unlike REAH are locally aggressive and is characterised by bone destruction and invasion to adjacent structures with a potential for malignant transformation. They occur almost exclusively on the lateral nasal wall and are unilateral. Histologically it is characterised by endophytically growing stratified squamous epithelium into sinonasal seromucinous glands. ${ }^{(3)}$

\section{Conclusion}

COREAH is an extremely rare benign hamartomatous lesion that can be clinically and radiologically mistaken for more common and other aggressive tumors of the head and neck. It is important to correctly identify these lesions to avoid unnecessary surgical intervention.

\section{References}

1. Fedda F, Boulos F, Sabri A. Chondroosseous respiratory epithelial adenomatoid hamartoma of the nasal cavity. Int Arch Otorhinolaryngol. 2013 Apr;17(2):218-21.

2. Chondro-osseous respiratory epithelial adenomatoid hamartoma of the nasal cavity (COREAH): A case report. J Otolaryngol-ENT Res [Internet]. 2019 Jun 3 [cited 2021 Jul 13]; Volume 11(Issue 3). Available from: https://medcraveonline.com/JOENTR/JOE NTR-11-00429.pdf

3. Daniel A, Wong E, Ho J, Singh N. Chondro-Osseous Respiratory Epithelial
Adenomatoid Hamartoma (COREAH): Case Report and Literature Review. Case Rep Otolaryngol. 2019 Jul 25;2019: 5247091. 